\title{
Les usages de la langue bretonne en politique
}

Uses of the Breton language in politics

Julien Meffre

\section{(2) OpenEdition}

\section{Journals}

Édition électronique

URL : https://journals.openedition.org/lbl/284

DOI : $10.4000 / / \mathrm{lbl} .284$

ISSN : 2727-9383

\section{Éditeur}

Université de Bretagne Occidentale - UBO

\section{Édition imprimée}

Date de publication : 1 mai 2017

Pagination : 85-109

ISBN : 979-10-92331-31-8

ISSN : $1270-2412$

\section{Référence électronique}

Julien Meffre, «Les usages de la langue bretonne en politique », La Bretagne Linguistique [En ligne], 21 | 2017, mis en ligne le 01 mai 2020, consulté le 23 mars 2022. URL : http://journals.openedition.org/lbl/ 284 ; DOl : https://doi.org/10.4000/lbl.284

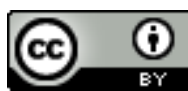

La Bretagne Linguistique est mise à disposition selon les termes de la Licence Creative Commons Attribution 4.0 International. 
Julien MEFFRE*

\section{Les usages de la langue bretonne en politique}

\section{Genèse et mise en œuvre d'une recherche en sociolinguistique}

A

l'origine de ce travail il y a un constat : la présence visible de la langue bretonne dans le paysage politique régional. À chaque élection locale, le breton s'invite dans le jeu électoral comme thème de campagne. Voici deux exemples pour illustrer ce phénomène. À Quimper, lors des élections municipales de mars 2014, la question de la défense et de la langue fait une entrée significative dans le débat public. Ainsi, au cours de la campagne, Ludovic Jolivet qui est élu à cette occasion maire de Quimper opère un revirement complet. Dans un premier temps, il se montre plutôt hostile à la promotion des langues régionales. Ainsi, pendant l'été 2013, il déclare, en soutien à Xavier Bertrand, que la Charte des langues régionales est « une litanie de mesures impossibles à appliquer ${ }^{1} »$. Suite à cette déclaration, il est en but à l'hostilité de certaines personnalités politiques régionalistes telles Léna Louarn et Naig Le Gars. Finalement, sous la pression de la gauche régionaliste, il s'engage concrètement à mettre en œuvre une politique linguistique. Il surenchérit même en promettant d'atteindre le niveau 4 de la Charte «Ya d'ar brezhoneg ». C’est une promesse électorale ambitieuse, alors

* Master 2 de breton à l’UBO.

1. Ouest-France: http://www.ouest-france.fr « breton du bech entre droite et gauche » (consulté le 1er mars 2014). 
que sa ville stagne au niveau 1 depuis la signature de la charte en 2008. Ce faisant, il se propose de faire de Quimper « la capitale de la culture bretonne ». Ce changement d'attitude est caractéristique du consensus apparent au sein de la classe politique régionale sur la question de la promotion et de la défense de la langue régionale.

Voici un autre exemple de la présence de la langue bretonne dans l'espace politique régional. À l'automne 2013, le slogan "Re zo re », cher au leader des Bonnets-Rouges Christian Troadec, fait écho jusque dans les médias nationaux ${ }^{2}$. Ce qui est au départ l'expression d'une révolte sociale et fiscale, face à l'essoufflement d'un modèle économique agricole déclinant, se mue rapidement en mouvement de protestation générale envers un État qualifié de jacobin par les leaders des Bonnetsrouges, Christian Troadec en tête. Rapidement, alors que le mouvement se politise, on observe une utilisation accrue des marqueurs identitaires régionaux ${ }^{3}$ au sein desquels le breton obtient une place de choix. Ainsi, le slogan « Bevañ, labourat ha divizout er vro», "Vivre, travailler et décider au pays » fait office de programme et s'inscrit résolument dans un sentiment régional revendiqué, sentiment dans lequel le breton à un rôle - a minima symbolique - à jouer. Ces deux exemples témoignent de la présence du breton dans l'espace politique régional.

Afin de confronter ces observations aux discours des acteurs politiques, j’ai décidé d'entreprendre l'édification d'un corpus composé d'entretiens réalisés avec des acteurs politiques bretonnants. Il est constitué d'entretiens dirigés et réalisés avec dix élus bretonnants et un non bretonnant. Le recueil de ces entretiens s'étale sur les deux années de travail dévolues à la production d'un mémoire de Master.

Mon premier axe de recherche a eu pour objet d'étudier ce que signifie, pour un représentant de l'État, le fait de s'exprimer dans une langue différente de la norme requise dans l'exercice du pouvoir en France (l'article 2 de la Constitution de la Cinquième République stipule que le français est la langue de la République). Il s'agit alors d'étu-

2. Louis MORICE, « Re zo re c'est trop pour les Bonnets Rouges, Le Nouvel Observateur, [en ligne], (consulté le 2 novembre 2013).

3. Lors des manifestations ainsi qu'aux assises du mouvement, le Gwenn ha du (drapeau breton) est omniprésent. 
dier quelles représentations de la langue bretonne ont l'homme et la femme politique qui s'expriment dans ou sur cette langue?

Une première lecture de ce corpus en cours d'élaboration me permet d'établir ce constat : le breton, qui était une langue d'usage en politique, principalement lors des campagnes électorales ${ }^{4}$, est devenu une langue dont l'usage est désormais essentiellement symbolique. En effet, alors que la sauvegarde du breton fait l'objet d'un consensus dans la classe politique régionale, cette langue n'est pratiquement jamais utilisée en tant que moyen de communication. Cet usage symbolique est présent à travers plusieurs occurrences. Le premier octobre 2013, le député régionaliste Paul Molac s'exprime en breton au sein du Palais Bourbon : "Comme on dit chez nous, pep tra zo mat a zo mat da gaout", "Tout ce qui est bon est bon à prendre », et voilà que le président de séance, Marc Le Fur, lui répond "matress tout an dud neus kie kompren » "peut-être que tout le monde n'a pas compris ». Ce qui peut avoir, pour le grand public (la séance est filmée), l'apparence d'une situation de communication en breton est en fait un échange purement symbolique.

En réalité, cette situation de communication est dès le départ vouée à l'échec. D'une part, seul quatre députés comprennent le breton dans l'hémicycle ${ }^{5}$. D'autre part, l'intercompréhension entre les deux hommes ne va pas de soi, ces deux députés usant d'une variété de breton sensiblement différente. De surcroît, M. Le Fur, se sentant peu à l'aise dans l'exercice, ne prend jamais la parole en breton en public.

Autre exemple : la place symbolique réservée au breton sur le matériel électoral. Ainsi, à l'occasion des élections départementales de mars 2015, sur la profession de foi des candidats de la majorité sortante du Conseil départemental, la contribution du PS à la promotion du breton se limite à la traduction du slogan de campagne général : «Holl asambles evit Penn ar Bed» [Tous ensemble pour le Finistère]. Quelques rubriques sont traduites en breton, mais l'effort de bilinguisme s'arrête là. C'est un affichage symbolique qui n'a pas vocation à être compris par le lecteur, ce passage traduit n'a pas une fonction de communication.

4. Je fais ici référence à mon corpus, avec le témoignage d'Yvon Abiven. Il utilisait le breton lors des tournées de porte à porte dans les villages.

5. Il s'agit des deux députés précités auxquels s'ajoutent M. Jean-Luc Bleunven, député de la troisième circonscription du Finistère et Annie Le Houérou, députée des Côtesd'Armor. 
Cette première problématique, qui fait le constat d'un usage essentiellement symbolique du breton en politique, est établie en parallèle à l'élaboration du corpus. C'est bien à partir de la construction de ce dernier que procède la recherche. Ensuite, j'ai constitué des groupes linguistiques afin de procéder à l'analyse de l'échantillon interrogé.

L'objet de cet article est d'étudier quels sont les critères qui président à la construction de l'échantillon. Dans un premier temps, on s'arrêtera sur la méthodologie et les écueils rencontrés pour ensuite évoquer la constitution des groupes linguistiques.

Tableau synthétique de l'échantillon

\begin{tabular}{|c|c|c|c|c|c|c|}
\hline Identité & $\begin{array}{l}\text { Date de } \\
\text { naissance }\end{array}$ & Profession & $\begin{array}{l}\text { Lieu de } \\
\text { naissance }\end{array}$ & $\begin{array}{l}\text { Lieu de } \\
\text { résidence }\end{array}$ & Mandats & Parti \\
\hline $\begin{array}{l}\text { Yvon } \\
\text { ABIVEN }\end{array}$ & $\begin{array}{l}1948 \\
\text { (67 ans) }\end{array}$ & $\begin{array}{l}\text { Enseignant } \\
\text { retraité }\end{array}$ & $\begin{array}{l}\text { Plounéour- } \\
\text { Trez }\end{array}$ & $\begin{array}{l}\text { Saint- } \\
\text { Thégonnec }\end{array}$ & $\begin{array}{l}\text { Maire de St-Thégonnec } \\
\text { (1989-2014). Conseiller } \\
\text { général (1992-2001). } \\
\text { Député (1997-2002) }\end{array}$ & $\begin{array}{l}\text { UDB puis } \\
\text { divers gauche }\end{array}$ \\
\hline $\begin{array}{l}\text { Jean-Luc } \\
\text { BLEUNVEN }\end{array}$ & $\begin{array}{l}1958 \\
\text { (56 ans) }\end{array}$ & Agriculteur & Plabennec & Plabennec & $\begin{array}{l}\text { Maire de Plabennec } \\
\text { (2008-2014). Député } \\
\text { élu en 2012 }\end{array}$ & $\begin{array}{l}\text { Divers } \\
\text { gauche. Parti } \\
\text { siège avec le } \\
\text { groupe } \\
\text { socialiste } \\
\text { à l'assemblée }\end{array}$ \\
\hline $\begin{array}{l}\text { Dominique } \\
\text { CAP }\end{array}$ & $\begin{array}{l}1967 \\
\text { (46 ans) }\end{array}$ & $\begin{array}{l}\text { Chargé de } \\
\text { mission au } \\
\text { PNRA }\end{array}$ & $\begin{array}{l}\text { Plougastel- } \\
\text { Daoulas }\end{array}$ & $\begin{array}{l}\text { Plougastel- } \\
\text { Daoulas }\end{array}$ & $\begin{array}{l}\text { Maire de Plougastel } \\
\text { depuis } 2001 \text { ( } 3^{\mathrm{e}} \text { mandat). } \\
\text { Vice-président de Brest } \\
\text { métropole }\end{array}$ & \\
\hline $\begin{array}{l}\text { Hervé } \\
\text { GUELOU }\end{array}$ & $\begin{array}{l}1961 \\
\text { (53 ans) }\end{array}$ & Agriculteur & Plufur & Plufur & $\begin{array}{l}\text { Maire de Plufur depuis } \\
1995 \text { ( } 4 \text { e mandat en } \\
\text { cours). Conseiller régio- } \\
\text { nal depuis } 2010 \text { (groupe } \\
\text { droite et centre) }\end{array}$ & \\
\hline $\begin{array}{l}\text { Didier } \\
\text { GOUBIL }\end{array}$ & $\begin{array}{l}1957 \\
\text { (58 ans) }\end{array}$ & Agriculteur & Poullaouen & Poullaouen & $\begin{array}{l}\text { Maire de Poullaouen } \\
\text { depuis } 2001 \text { ( } 3^{e} \text { mandat } \\
\text { en cours). Premier } \\
\text { vice-président de Poher } \\
\text { communauté }\end{array}$ & \\
\hline $\begin{array}{l}\text { Gérard } \\
\text { LAHELLEC }\end{array}$ & $\begin{array}{l}1954 \\
\text { (61 ans) }\end{array}$ & Technicien & Plufur & Plufur & $\begin{array}{l}\text { Vice-président du } \\
\text { Conseil régional en } \\
\text { charge des transports, } \\
\text { élu en 2004. Candidat à } \\
\text { un } 3^{\mathrm{e}} \text { mandat }\end{array}$ & $\begin{array}{l}\text { Parti } \\
\text { communiste } \\
\text { français }\end{array}$ \\
\hline $\begin{array}{l}\text { Félix } \\
\text { LEYZOUR }\end{array}$ & $\begin{array}{l}1932 \\
\text { (82 ans) }\end{array}$ & $\begin{array}{l}\text { Enseignant } \\
\text { retraité }\end{array}$ & Plourac'h & Plourac'h & $\begin{array}{l}\text { Maire de Callac } \\
\text { (1983-2008). Conseiller } \\
\text { général (1970-2008). } \\
\text { Sénateur (1989-1997). } \\
\text { Député (1997-2002) }\end{array}$ & $\begin{array}{l}\text { Parti } \\
\text { communiste } \\
\text { français }\end{array}$ \\
\hline
\end{tabular}




\begin{tabular}{|l|l|l|l|l|l|l|}
\hline $\begin{array}{l}\text { Lena } \\
\text { LOUARN }\end{array}$ & $\begin{array}{l}1950 \\
(64 \text { ans })\end{array}$ & $\begin{array}{l}\text { Respon- } \\
\text { sable } \\
\text { associatif }\end{array}$ & $\begin{array}{l}\text { Villeneuve- } \\
\text { Saint- } \\
\text { Georges }\end{array}$ & Rennes & $\begin{array}{l}3^{e} \text { Vice-présidente du } \\
\text { Conseil régional en } \\
\text { charge des langues de } \\
\text { Bretagne }\end{array}$ & \\
\hline $\begin{array}{l}\text { Paul } \\
\text { MOLAC }\end{array}$ & $\begin{array}{l}1962 \\
(52 \text { ans })\end{array}$ & $\begin{array}{l}\text { Professeur } \\
\text { du } \\
\text { secondaire }\end{array}$ & Ploermel & Ploermel & $\begin{array}{l}\text { Député du Morbihan } \\
\text { élu en 2012 }\end{array}$ & $\begin{array}{l}\text { Siège à } \\
\text { l'Assemblée } \\
\text { avec le } \\
\text { groupe écolo- } \\
\text { giste, proche } \\
\text { de l'UDB }\end{array}$ \\
\hline $\begin{array}{l}\text { Marie- } \\
\text { Claude } \\
\text { MORVAN }\end{array}$ & $\begin{array}{l}1955 \\
(58 \text { ans })\end{array}$ & Agricultrice & Hanvec & Hanvec & $\begin{array}{l}\text { Maire de Hanvec depuis } \\
\text { 2001. Vice-présidence } \\
\text { de la Communauté de } \\
\text { commune }\end{array}$ & $\begin{array}{l}\text { Divers } \\
\text { gauche }\end{array}$ \\
\hline $\begin{array}{l}\text { Christian } \\
\text { TROADEC }\end{array}$ & $\begin{array}{l}1966 \\
(49 \text { ans })\end{array}$ & $\begin{array}{l}\text { Chef } \\
\text { d'entreprise }\end{array}$ & Carhaix & Carhaix & $\begin{array}{l}\text { Maire de Carhaix depuis } \\
\text { 2001 (3e mandat en } \\
\text { cours). Président de } \\
\text { Poher communauté. } \\
\text { Conseiller régional } \\
\text { (2004-2010) }\end{array}$ & $\begin{array}{l}\text { Divers } \\
\text { gauche. } \\
\text { Régionaliste : } \\
\text { conseiller } \\
\text { régional } \\
\text { apparenté } \\
\text { UDB. Proche } \\
\text { du Parti } \\
\text { breton }\end{array}$ \\
\hline
\end{tabular}

\section{Méthodologie}

La méthodologie s'appuie sur une enquête de terrain reposant sur la réalisation d'entretiens ethnographiques. J'ai immédiatement choisi d'aborder mon enquête de terrain par le biais de la méthode qualitative. Tout en gardant bien à l'esprit que le chercheur doit objectiver son sujet, il m'est apparu très important d'aller recueillir la parole d'élus sur le terrain.

Cela m'a permis d'accumuler des données que je n'aurais pu obtenir via un questionnaire. En outre, l'environnement et l'ambiance dans lesquels se sont déroulés les entretiens participent également de l'explication sociolinguistique.

En des termes plus scientifiques, j’ai essayé d'appliquer les préceptes de la méthode "empirico-inductive » développée par le chercheur en sociolinguistique Philippe Blanchet ${ }^{6}$.

Suivant cette méthode, je me suis efforcé de prendre en compte le contexte et l'histoire de chacun des témoins observés-interrogés.

6. Philippe BlANCHET, La linguistique de terrain, méthode et théorie, une approche ethnolinguistique de la complexité, Rennes, PUR, 2000, p. 33. 
Le «système de référence $»^{7}$ et le passé de chaque individu, sont des données importantes pour saisir un témoin dans sa complexité. Afin de mieux prendre en compte ces aspects, j'ai établi la biographie linguistique et politique de chaque personne interrogée. Celle-ci éclaire le rapport de chaque témoin avec mon sujet : la langue bretonne, dans un contexte déterminé - discours ou pratique politique - pour quel usage (politique ou non), dans quel contexte et avec quelles représentations. Enfin, je dois prendre en compte les interactions, l'effet que je produis sur mon interlocuteur : cet aspect est ici fondamental, car s'exprimer en langue bretonne, aujourd'hui, n'est pas anodin. Cela peut produire des phénomènes de convivialité et de proximité (c'est bien ce que j'ai ressenti dans la plupart de mes entretiens) ou a contrario des sentiments d'insécurité linguistique peuvent apparaître. Bien évidemment, cela peut nuire considérablement à la qualité de l'entretien si le témoin est mis en difficulté par une maitrise approximative de la langue. Une telle méthode implique des allers-retours entre le terrain (ici les enquêtes) et le travail théorique. C'est ainsi que j’ai procédé, et, si les premiers entretiens produisirent des résultats plus aléatoires, ma réflexion s'est enrichie peu à peu d'aspects théoriques dont je n'avais pas connaissance au début de l'enquête. Il faut préciser toutefois, que je n'ai pas suivi, stricto sensu, la méthode dite du « sablier» de Philippe Blanchet. J'ai éludé la première partie qui préconise l'observation participante. Néanmoins, j'ai gardé à l'esprit l'idée que l'intuition du chercheur ne suffit pas et qu'il faut toujours fonder son travail sur des enquêtes ${ }^{8}$.

\section{Des entretiens semi-directifs}

Il s'agit bien ici de questions ouvertes favorisant une parole libre. Dans une perspective interactionniste, j'ai posé quelques questions, toujours ouvertes, qui permettaient le libre déroulement de la pensée. Si le témoin s'éloignait du sujet, je n'ai pas cherché à le corriger. Ces questionnaires furent expérimentés in vivo et je dois mentionner qu'au début de ma recherche, je n'avais pas les armes conceptuelles du sociolinguiste débutant. Les deux premiers entretiens peuvent ainsi être qualifiés d'exploratoires. C'est pourquoi je n'ai pas réalisé de guide

7. Ibidem, p. 34.

8. Ibid., p. 40. 
d'entretien préalable. Par la suite, j'ai modifié certaines questions, ayant pris conscience de la perfectibilité de quelques éléments. C'est bien en croisant la recherche entre un corpus théorique et bibliographique et les enquêtes de terrain que je suis parvenu à améliorer mon terrain.

\section{Remarques sur le déroulement de l'enquête de terrain}

Si la prise de contact préalable aux entretiens est souvent considérée comme décisive ${ }^{9}$, l'enquête que j'ai réalisée présente une originalité marquée qui résulte des fonctions occupées par mes témoins. Hormis Félix Leyzour, qui est désormais retiré de la vie politique, j’ai toujours eu affaire à un intermédiaire lors de la prise de rendez-vous, c'était bien souvent le ou la secrétaire de mairie.

Dans un premier cas de figure, je relève quelques interactions préalables à l'entretien. D'abord avec Félix Leyzour que j'ai rencontré et sollicité de manière informelle, puis avec Yvon Abiven qui me rappela afin de convenir d'un rendez-vous. Marie-Claude Morvan, maire de Hanvec fit de même, mais cela est anecdotique si l'on considère que je la connais depuis longtemps.

Dans un deuxième cas de figure, je fis la connaissance de mes témoins le jour de l'entretien. Cela ne me permit pas de deviner si mon interlocuteur était bien disposé à répondre à une telle sollicitation. Il faut souligner qu'il est assez aisé d'obtenir l'accord de personnalités publiques ayant l'habitude de s'exprimer dans les médias. A fortiori s'il ne s'agit pas d'un thème polémique. J'ai systématiquement présenté mon sujet de manière évasive, livrant seulement les deux mots-clés que sont ici « politique et breton ». Je dois préciser que ce type de témoin présente des dispositions favorables quant à l'enregistrement de sa parole.

Le lieu de l'entretien s'est imposé de lui-même. Il se confond souvent avec le lieu d'exercice du pouvoir : la mairie, le bureau du Conseil régional, ou encore la permanence pour le député Jean-Luc Bleunven. Néanmoins, à trois occasions, j’ai mené mon entretien au domicile de mon témoin, dans la cuisine ou le bureau. La distanciation requise dans le cadre d'un travail de recherche peut s'avérer moindre dans un tel décor. Sachant que le cadre de l'entrevue peut influer sur le témoin, il importe de bien le choisir. Les mairies de campagne affichent

9. Frédéric GonTHIER, «Conduire un entretien : les quatre temps », dans P. Bréchon (dir.), Enquêtes qualitatives enquêtes quantitatives, Grenoble, Presses universitaires de Grenoble, 2011, p. 53. 
souvent un air de solennité et de pompe républicaine. Le bureau du maire, orné du portrait du président de la République, accentue le caractère immuable du décorum. Dès que la ville atteint une taille respectable, le bureau paraît vaste et confortable. C'est ainsi que je classe volontiers les villes de Plougastel et Carhaix dans cette catégorie.

On chercherait en vain dans ces locaux l'existence de la langue bretonne, elle en est totalement absente.

La nature particulière du public visé a engendré une autre difficulté d'ordre matériel. Le cumul des mandats est une spécialité française. La traduction de tout ceci sur le terrain est que le maire d'une commune rurale occupe toujours une fonction à l'échelon intercommunal. Ainsi, il est souvent en proie à un emploi du temps surchargé. La durée d'entretien minimum d'une heure suggérée dans les manuels de sociologie est difficile à respecter. Il n'est pas toujours évident d'obtenir le consentement d'une personne débordée sur la base d'un entretien si long. J'ai eu parfois de bonnes surprises. Ainsi, mon entrevue avec la conseillère régionale Lena Louarn dura une heure. Elle a pourtant la réputation (que je ne crois pas usurpée) d'abattre un travail considérable et ne dispose pas de beaucoup de temps. À l'évidence, il faut toujours prévoir une amplitude horaire large.

Au début de chaque entretien, il m'a fallu en expliciter le thème général, il s'agit ici d'énoncer la consigne. La problématique n’a pas à être formulée sinon on s'expose au risque d'une prise de position du témoin quant à celle-ci ${ }^{10}$. Pour conserver une certaine liberté de parole, il ne faut pas donner au témoin le sentiment d'une réification.

Au cours de certains entretiens j'ai dû faire face à un problème bien connu des sociologues. Selon la formule de Janine Barbot ${ }^{11}$, " confronté à une tension majeure entre laisser l'enquêté explorer à sa guise le thème qui lui est proposé et le recadrer s'il semble trop s'en écarter », on est souvent tenté de laisser libre cours aux digressions de son interlocuteur.

De plus, j'ai souvent été confronté à des personnalités singulières. Celle de Félix Leyzour se caractérise par sa truculence. Christian Troadec déroule un discours politique extrêmement maitrisé sur un ton

10. Ibid., p. 53.

11. Janine BARBOT, « Mener un entretien de face à face », dans S. Paugam (dir.), L'enquête sociologique, Paris, PUF, 2012, p. 116. 
très familier. Lena Louarn se montre très volubile et sûre de son fait. Yvon Abiven aime raconter sa longue carrière politique. Gérard Lahellec, fidèle à sa réputation, s'exprime avec emphase. La nature particulière de l'échantillon se traduit dans le fait que j'ai eu affaire dans la plupart des cas à des professionnels de la parole. Quoi qu'il en soit, avec des acteurs politiques présentant de telles aptitudes à la mise en scène de soi, il faut garder à l'esprit que le discours est toujours construit. Souvent, une conversation anodine cache un discours politique bien rodé. Toutefois, il ne faut pas tirer de conclusions hâtives. Tous ces élus ne sont pas des « communicants » chevronnés. La nature publique de l'échantillon singularise considérablement mon travail et l'éloigne en partie d'une enquête sociologique traditionnelle. En effet, nul besoin d'anonymer l'échantillon ici. Les élus parlent à visage découvert et la restitution de mon travail peut s'envisager sans crainte.

\section{Des biographies linguistiques}

J'ai décidé de réaliser la biographie linguistique et politique de chacun de mes témoins afin de comprendre dans quelle mesure ces éléments participent de leur vision de la langue bretonne.

J'y ai inclus une description de l'entretien dans une perspective interactionniste. Voici un exemple de fiche :

\section{Fiche signalétique de Félix Leyzour}

Nom, prénom : Leyzour, Félix

Année et lieu de naissance : né en 1932 à Plourac'h, 81 ans au moment de l'entretien

Profession : instituteur à la retraite

Lieu d'exercice du mandat: Callac, Saint-Brieuc, Paris

Mandats : Conseiller général du canton de Callac 1970-2008, Maire de Callac : 1983-2008, Sénateur 1989-1997, Député 1997-2002

\section{Langue bretonne}

Apprentissage : Félix Leyzour a comme langue première le breton. Il a appris le français à l'école primaire.

Pratique : Mr Leyzour s'exprime dans son parler vernaculaire de la région de $\mathrm{Callac}^{12}$. Il ne possède pas de registre linguistique plus élevé

12. Je développe ce point dans la partie dédiée aux groupes linguistiques. 
en breton. Quand il souhaite utiliser un registre de langue disparitaire, il utilise le français, langue qu'il manie parfaitement, en bon instituteur formé à l'École Normale. À noter, le recours systématique au français dès lors que l'on aborde le champ lexical de la politique ou quand il faut employer des termes techniques. Manifestement, l'ancien sénateur ne connait pas les derniers développements lexicaux suscités par l'Office public de la langue bretonne.

\section{Entretien}

Date de l'entretien : 21 octobre 2013 Durée : environ 41 minutes Déroulement de l'entretien : ma rencontre avec Félix Leyzour s'est déroulée de façon fortuite et amusante. Je l'ai croisé une première fois lors d'un «repas de rue » à Callac pendant l'été 2013. Le décorum est assez pittoresque. On sert une nourriture roborative à base de cochon et pommes de terre, le groupe les Ramoneurs de Menhir fait résonner des riffs stridents émaillés de couplets séparatistes. J’aperçois les frères Morvan, pas encore affublés de bonnets ${ }^{13}$, qui déambulent tranquillement dans la foule joyeuse. Au milieu d'une foule assez représentative de ce coin reculé du Centre-Bretagne (rurale, modeste et de tout âge), j’aperçois un monsieur bien mis duquel se dégage une certaine aura de solennité. Ainsi qu'un vernis de notabilité. Une bonhomie tranquille vous donne immédiatement envie d'aller à sa rencontre. Ce que je m'empresse de faire aussitôt l'ayant entendu parler breton. Il me demande, tout de go, si je suis journaliste et je pense que cela me signale à l'évidence comme un « horsain ». La conversation s'élance tantôt en français, tantôt en breton. Rapidement, il m'apprend qu'il a été le premier édile de la cité où nous nous trouvons. Ravi de l'aubaine, je lui demande s'il serait possible que l'on se rencontre pour un entretien. Il se fait prier, mais finit par me donner l'autorisation de l'appeler à son domicile afin de fixer ce rendez-vous. Deux mois et demi plus tard, je l'appelle et il accepte volontiers. Anecdote révélatrice, il me propose comme lieu de rencontre la mairie, dont il n'est plus à la tête depuis cinq ans. Il faut savoir, qu'au mois de novembre 2013, c'est Carole Le Jeune qui est maire de la cité. Elle a fait partie de l'équipe municipale de Félix Leyzour pendant treize ans. L’ex-sénateur est à la mairie en terrain ami. Finalement, M. Leyzour préfère que l'entrevue se tienne à son domicile, dans son cabinet de travail-bibliothèque.

D’emblée il me tutoie, comme il l'avait déjà fait lors de notre première rencontre. Je ne distingue pas bien dans quelle mesure cela révèle 
un politique chevronné, ou alors l'homme de terroir. Je précise que l'utilisation du vouvoiement dans le parler local ${ }^{14}$ est rare. Le discours est bien rodé et ponctué d'anecdotes à caractère parfois linguistique. Félix Leyzour est un bon client, c'est un personnage truculent, qui ne fait pas de détours quand il s'agit d'exprimer ses vues personnelles.

Le chercheur débutant que je suis est sans doute trop en empathie avec ce personnage haut en couleur. Il faudrait opérer une certaine distanciation et objectivation du sujet.

Le but de ces biographies linguistiques est d'éclairer le rapport des témoins avec le sujet afin de mieux saisir le rapport que chacun entretient avec la langue bretonne. Comme l'explique le sociologue Michel Pialoux, « seul l'analyse du contexte d'entretien permet de donner tout son sens aux propos tenus par les enquêtés ». Ainsi, les analyses des interactions enquêteurs-enquêtés participent de la construction du sujet.

Le caractère singulier des interactions se trouve renforcé par le biais de la langue de l'entretien : le breton. Généralement, cela produit des phénomènes de proximité et de convivialité. C'est d'autant plus vrai si l'on considère que les témoins ne possèdent pas de variétés distinguées de breton. A contrario, un sentiment d'insécurité linguistique est venu perturber l'entretien avec Jean-Luc Bleunven. Son breton est assez hésitant et cela a altéré la qualité de notre entretien. Néanmoins, c'est un phénomène éclairant. Ainsi, M. Bleunven témoigne de la difficulté pour lui de conduire les affaires politiques en breton, il explique na pas être assez à son aise pour parler breton en public. Cela peut sembler paradoxal si l'on considère que le député de la troisième circonscription du Finistère est très régulièrement invité dans les médias bretonnants.

\section{La composition de l'échantillon et le choix des témoins}

J'ai fait le choix de rencontrer des personnalités politiques que j'avais eu l'occasion d'entendre à la radio ou de voir à la télévision. L'échantillon comporte onze témoins et présente deux femmes pour neuf hommes. La parité, objectif affiché et revendiqué par la gauche gouvernementale, est loin d'être une réalité sur le terrain, en particulier lorsque l'on recherche des bretonnants.

14. Vérification faite auprès de Francis Favereau : il est extrêmement rare de vouvoyer son interlocuteur dans ce secteur qu'il connait bien. 


\section{Le mandat}

Le postulat de départ de ma recherche met en évidence que, si la langue bretonne n'est plus que rarement une langue d'usage, on peut toutefois l'entendre quotidiennement dans un espace médiatique dédié. Cet espace est essentiellement radiophonique et associatif. Les médias financés par l'État font également, dans une moindre mesure, entendre la langue bretonne ${ }^{15}$.

On sait que le breton est une langue territorialisée (la BasseBretagne) et que la majorité de ses locuteurs est âgée ${ }^{16}$ et habite des espaces ruraux. Alors, on ne s'étonnera pas que les bretonnants soient bien représentés au sein des conseils municipaux de communes rurales.

Nous sommes là dans la sphère de la politique locale. Le champ médiatique privilégié où s'entend la voix de ces élus relève également de l'échelon local (radios) ou régional (télévision). Je fais alors le constat suivant : la figure du maire d'une commune rurale incarne un agent social majeur dans la communauté linguistique bretonnante. C'est ainsi, que dans mon échantillon, on trouve huit témoins sur onze ayant occupé la fonction mayorale.

Monsieur ou madame le maire est «Le personnage politique le plus populaire dans l'opinion » selon Hugues Portelli ${ }^{17}$. Il est, quand on parle de politique locale, une figure incontournable. Marie-Claude Morvan compare volontiers sa fonction à celle du curé, personnage désormais absent de la vie communale ${ }^{18}$. La fonction est ancienne et bien identifiée, elle existait déjà bien avant la Révolution. Elle jouit encore d'un pouvoir d'attraction important comme en témoigne la longévité exceptionnelle dans l'occupation des mandats. Ce phénomène est particulièrement remarquable dans les communes rurales ${ }^{19}$. Sur ce point mon échantillon ne fait pas exception à la règle : le seul témoin à n'avoir fait qu'un seul mandat de maire est Jean-Luc Bleunven. Quand, en 2012, il est élu député de la circonscription « Brest Rural », il décide

15. Il s'agit de France Bleu Breizh Izel et France 3 Bretagne.

$16.70 \%$ ont plus de 60 ans, cf. en introduction.

17. Hugues PORTELLI, «Les fondements administratifs d'un pouvoir politique », Pouvoirs, $\mathrm{n}^{\circ} 148,2014$, p. 5.

18. Marie-Claude MoRvan, "Hañveg : merhed en ti-kêr a-raog leh all», Brud Nevez, $n^{\circ} 303,2014$, p. 5.

19. H. PORTELLI, op. cit., p. 10. 
de ne pas se représenter au poste de maire à Plabennec. Par là même, il affirme être en faveur du non-cumul des mandats. Le reste de mon échantillon présente un minimum de trois mandats effectués. À cet égard, la longévité des mandats de MM. Guélou, Leyzour et Abiven (quatre mandats) à la tête de leurs mairies respectives est remarquable. Le phénomène semble perdurer avec la nouvelle génération. L'examen des situations de deux maires de mon échantillon le prouve. Ainsi, MM. Troadec, Guélou et Cap se sont présentés jeunes à la fonction mayorale (autour de 35 ans). Mais cela ne les empêche pas de solliciter et d'obtenir un quatrième mandat auprès de leurs électeurs ${ }^{20}$. La longévité du mandat de maire est exceptionnelle et présente des avantages non négligeables : «Cette longévité est source d’influence irremplaçable pour l'élu qui se trouve à la tête d'un capital de réseaux, liens personnels et d'expérience ${ }^{21}$. » Il arrive qu'un maire rural, par ses actions ou son charisme, en vienne à incarner sa commune. Je pense à Alfonse Arzel, ancien maire de Ploudalmézeau. Bien évidemment, un ancrage local ne peut desservir pour les échéances électorales. Le fait de parler l’idiome local est un atout qu'on ne saurait cacher ; c'est un trait distinctif qui inscrit l'élu dans une communauté sociale et linguistique ${ }^{22}$.

Tous ces éléments expliquent une surreprésentation des maires dans mon échantillon. Néanmoins, j'ai cherché à m'entretenir avec des élus ayant exercé des mandats nationaux pour chercher comprendre si une fonction supérieure changerait le regard sur la langue. C'est pourquoi on relève la présence de trois (ex) députés (MM. Abiven, Bleunven et Leyzour) au sein de mon échantillon.

\section{La couleur politique}

L'examen de mon échantillon fait apparaître une surreprésentation des élus de gauche ou apparenté. Sur un panel de onze acteurs politiques, huit peuvent être classés à gauche. Fait remarquable, aucun d'entre eux n'est membre du Parti Socialiste. Jean-Luc Bleunven siège avec le groupe socialiste à l'Assemblée nationale mais il n'est pas en-

20. Tous deux furent réélus au premier tour du scrutin municipal du 23 mars 2014.

Dominique Cap avec $51 \%$ des voix, $66 \%$ pour Christian Troadec.

21. H. Portelli, op. cit., p. 10.

22. William LABOV, Sociolinguistique, Paris, Éditions de Minuit, 1976. 
carté au Parti socialiste. En creux, cela révèle une forte représentation, au sein de mon échantillon, de la composante régionaliste du paysage politique breton. Est-ce la résultante d'un manque de représentativité du groupe étudié ? Cela donne-t-il une image fidèle des locuteurs de breton existant parmi les hommes et femmes politiques de la région? Deux réponses possibles à cette double interrogation. D'une part, selon les dires de M. Cap, qui me furent confirmés par Fañch Broudic ${ }^{23}$ après mon intervention lors du colloque à l'origine de cet article, il y a numériquement moins de représentants de la droite capables de s'exprimer en breton que de responsables de l'autre bord. D'autre part, le caractère restreint de l'échantillon ne me permet pas de prétendre à une quelconque ambition d'exhaustivité. Néanmoins, il est incontestable que la gauche régionaliste est très bien représentée dans mon échantillon et on peut inclure quatre personnalités dans ce groupe : Yvon Abiven, Lena Louarn, Paul Molac et Christian Troadec. Ce groupe se singularise par des représentations positives de la langue bretonne et un soutien sans faille aux glottopolitiques.

De facto, les représentations positives que ces acteurs ont du breton sont liées à leur inscription au cœur du mouvement régionaliste, bien plus qu'à une appartenance à la gauche ${ }^{24}$. Le clivage droite-gauche est assez inopérant pour l'analyse de l'échantillon. Il faut saisir l'évolution historique du mouvement régionaliste breton suite au tournant de Mai $68^{25}$ pour comprendre cette surreprésentation idéologique de la gauche régionaliste dans mon échantillon.

\section{La constitution de groupes linguistiques}

«Dans toutes les communautés linguistiques, il existe des personnes réputées mieux parler leur langue que d'autres ${ }^{26}$.»

23. En tant que responsable des programmes en breton de France 3 Bretagne, il a interrogé quasiment tous les responsables politiques bretonnants.

24. Julien MEFFre, Les usages du breton en politique, Mémoire de Master 2, Brest, UBO, 2015.

25. Vincent PORHEL, « La construction du discours régionaliste en Bretagne dans les années 1968 au travers de cinq conflits sociaux (1966-1981)», dans R. Le Coadic (dir.), Identités et sociétés de Plougastel à Okinawa, Rennes, p. 288.

26. Michel BERT, Colette GRINEVALD (dir.), «Linguistique de terrain sur langues en danger, Locuteurs et linguistes », Faits de Langues, n 35-36, Ophrys, 2010. 
Le breton, à l'évidence, n'échappe pas à la règle. En outre, pour les chercheurs spécialistes des langues en danger que sont Michel Bert et Colette Grineval, cette typologie de langue présente des spécificités. La première caractéristique de ces communautés linguistiques est l'absence de monolingues et « une forte proportion de bilingues aux compétences linguistiques variables ». La deuxième permet de relever une profusion de profils linguistiques atypiques. D'où l'importance d'établir une typologie précise des locuteurs de ces langues en péril.

Alors, je m’applique ici à établir une typologie des différents groupes composant la communauté linguistique bretonnante.

\section{Les locuteurs de tradition}

J'entends par là éviter l'emploi de l'expression «bretonnant de naissance », car elle définit mal le breton parlé par cette frange de locuteurs, encore majoritaire aujourd'hui. Ces locuteurs usent de formes dialectales, de parlers locaux dont la principale caractéristique est leur endogénéité ${ }^{27}$. À l'inverse, Lena Louarn ou Paul Molac parlent un néobreton assez éloigné des parlers locaux. Mme Louarn a reçu comme langue première de socialisation cet idiome particulier pour ensuite le transmettre à ses enfants. Naturellement, ils se perçoivent comme «bretonnants de naissance ». Cependant, tant en ce qui concerne la phonologie, la syntaxe, ou le lexique, leur breton est très différent de celui des parlers endogènes.

Les locuteurs de tradition sont les plus nombreux au sein de mon échantillon. Il est donc représentatif de l'ensemble des locuteurs quant à ce critère. Félix Leyzour, Gérard Lahellec, Marie-Claude Morvan et Hervé Guélou s’inscrivent dans ce groupe.

Pour le premier de mes témoins dans cette catégorie, M. Leyzour, l'âge est le facteur déterminant. Il est né au début des années trente au sein d'une communauté rurale où la langue d'usage est très majoritairement le breton. Comme beaucoup de locuteurs nés avant la Seconde Guerre mondiale, il ne parle pas un mot de français lorsqu'il fait son entrée à l'école. Son breton, comme l'atteste des traits linguistiques remarquables, est typique de la région de Callac.

27. E. LE PIPEC, op. cit., p. 104. 
Voici quelques exemples établissant le caractère endogène du parler de M. Leyzour :

- l'utilisation de ['pae:Rus] pour désigner la paroisse est attestée dans le NALBB ${ }^{28}$ au point 77, non loin de Callac. Ce point correspond à la commune de Saint-Servais à environ six kilomètres de Callac. C'est cette forme que l'on retrouve dans la transcription à la ligne 52 .

- l'occurrence "peut-être »: on relève [ma'tRen] à Saint-Servais comme dans la retranscription (1.58 et 1.142) provenant de mon entretien avec l'ancien sénateur.

- l'occurrence «quelque chose »: au point 77 , on relève [n dRa 'mnkkət], ce qui est proche de ce qu'emploie Félix Leyzour 1.192.

- l'occurrence «toujours»: donne [deh'ma:t] au point $77 \mathrm{du}$ NALBB, forme similaire (1. 146 et 1. 248) à celle utilisée dans le parler de l'ancien maire de Callac.

- l'idiomatisme : «il y a » on trouve [be zo] à Saint-Servais, forme très proche de celle employée par Me Leyzour (1.88) dans la transcription.

- Ou encore « celui-ci »: [hę:s] que l'on retrouve à l'identique dans ma transcription : 1.100 et 1.101 .

Je note avec regret que le NALBB ne donne pas les paradigmes du verbe être. J'ai donc eu recours à l'atlas linguistique de Pierre Le Roux ${ }^{29}$. Dans ce dernier, le point le plus proche de Callac se trouve être la commune de Lohuec : au point 21. Douze kilomètres séparent les deux localités. C'est conséquent en dialectologie, domaine de la microvariation. Pour certains termes cela peut être acceptable si on peut tracer une isoglosse assez large.

- «tu as » est noté [fœs] dans l'atlas, c'est une forme proche de celle que je relève dans ma transcription : 1.89 et 91 .

28. Jean LE DÛ, Nouvel Atlas Linguistique de la Basse-Bretagne, Brest, CRBC, 2001. Noté ici NALBB.

29. Pierre Le Roux, Atlas linguistique de la Basse-Bretagne, Rennes, Plihon et Hommay, 19241963. 


\section{Les semi-locuteurs}

Le terme semi-locuteur est la traduction française de l'expression semi speaker proposée par Michel Bert ${ }^{30}$.

Cette expression tente de définir ces locuteurs au niveau de compétence linguistique plus faible que ceux du groupe précédent. Cette frange de locuteurs n'a appris que partiellement la langue ou l'a oubliée en partie. Nancy Dorian, qui travaillait sur le gaélique d'Écosse, fut la première chercheuse à isoler ce type de locuteur possédant une compétence partielle dans une langue menacée.

Selon ce chercheur, l'existence d'une telle catégorie de locuteurs est caractéristique de ce type de langue. Cette catégorie de locuteurs possède « des compétences complètes en réception ». C'est la production qui les distingue. Certains sont capables de s'exprimer correctement quand d'autres peinent à le faire ${ }^{31}$. C'est souvent une pratique irrégulière de la langue qui distingue ces locuteurs de ceux de la première catégorie. Cette assertion se vérifie pour Dominique Cap et Jean-Luc Bleunven, et dans une moindre mesure pour Didier Goubil.

Dominique Cap et Didier Goubil maitrisent suffisamment la langue bretonne pour tenir une conversation sans trop d'interruptions. Mais leur compétence linguistique les place bien dans le type semilocuteurs. En effet, on relève chez tous deux d'importantes fautes grammaticales (notamment les mutations consonantiques) qui prouvent une acquisition imparfaite de la langue ou un manque de pratique.

Le cas de Jean-Luc Bleuven est plus épineux. Il illustre la difficulté d'établir une typologie très fine de locuteur. Par certains aspects, le breton de M. Bleunven est celui d'un terminal speaker, mais il est tout de même en mesure de produire un discours en breton. C'est pourquoi je l'ai rattaché aux groupes des semi-locuteurs. Une analyse rapide de notre entretien met en lumière une compétence linguistique moyenne. Le discours est ponctué d'hésitations, de coupures et de mots français. Par exemple, il ne comprend pas ma première question «E peseurt-mod e rit gant ar brezhoneg e-pad ho pubezpolitik? " [Comment utilisez-vous le breton pendant vous activités politiques] je pense qu'il ne saisit pas la tournure peseurt mod. Il aurait été préférable que j’utilise penaos. En

30. Michel BerT, Colette Grinevald, op. cit., p. 122.

31. Ibidem, p. 126. 
outre, le député Bleunven cherche constamment ses mots. Il déclare souffrir d' un manque de lexique pour s'exprimer en breton sur des sujets connexes à son activité politique (1.17). Cela se traduit par un sentiment d'insécurité linguistique qu'il avoue spontanément et sans aucune gêne. Ainsi, il explique ne pas prendre la parole en public : «n'on eas awalac'b gant ar brezoneg»: [je ne suis pas assez facile avec le breton]. La traduction littérale est sibylline, maladroite dans sa formulation et elle démontre une maitrise imparfaite de la langue.

Le regard qu'il porte sur sa langue n'est pas empreint d'un sentiment d'infériorité, comme souvent chez les bretonnants de tradition. Il a même engagé comme assistante parlementaire une bretonnante, afin de faire la part belle au breton sur son site personnel. L'absence de sentiment d'infériorité peut s'expliquer par l'assurance que lui procure une bonne maitrise du français, la langue dominante.

Je relève des marques dialectales dans le breton parlé par JeanLuc Bleunven. Elles sont moins nettes que dans le cas des locuteurs traditionnels. Une acquisition parcellaire de la langue et un lexique appauvri en sont la conséquence. Par exemple, M. Bleunven utilise toujours le possessif va (1.8), il prononce la diphtongue [eas] pour aes (1.24). «Pour moi » est prononcé [evidoun].

Une analyse rapide du discours de Dominique Cap fait apparaître les mêmes phénomènes linguistiques. On observe chez le maire de Plougastel beaucoup de fautes de mutations consonantiques. Par exemple: «leun a traoù, [eu], kalz a tud» 1.5 de l'entretien. Il cherche souvent ses mots, hésite, phénomène qui est à son comble quand il cherche à signifier qu'un grand nombre d'enfants est scolarisé en breton dans sa commune : "Plougastel amañ ez e [...], [eu], ouspenn [eu], pemp, pemp war'nugent [eu], pour cent hag e re yaouank a zo o teskin brezoneg, setu ze ar barrez, hag ez. eus [...], meur a [aaa] ar re yaounk, hag a zo o teski breqoneg e Breiž, [À Plougastel, ici, plus de cinq, vingt-cinq, pour cent, des jeunes qui apprennent le breton, voilà la paroisse dans laquelle il y a le plus de jeunes en train d'apprendre le breton en Bretagne].

Je perçois également des phénomènes d'hypercorrections : «bum, ya, meu, ap, ablamour zoun, [eu] e gojean brezhoneg ». Mais si l'on compare attentivement les deux transcriptions, le maire de Plougastel est plus à son aise en breton que le député Jean-Luc Bleunven. Le discours qu'il porte sur sa langue est très positif, il fait état d'un apprentissage par 
transmission familiale qu'il oppose au breton appris dans les livres : «Setu, va brezoneg, n'eo ket brezoneg al leurioù, me brezoneg ar mêz.»: [voilà, le breton que j'ai appris, n'est pas celui des livres mais celui des champs]. Il assure parler en breton avec ses administrés bretonnants de tradition. Cela lui assure une pratique minimale et lui permet de s'exprimer correctement. Il est toutefois amusant de constater une absence de regard critique sur une langue émaillée de fautes.

\section{Des locuteurs du néo-breton}

Si les chercheurs s'accordent en général sur l'existence d'un «néobreton ${ }^{32}$ ", appelé parfois « breton standard » ou « breton littéraire », il est difficile d'établir une typologie précise des locuteurs de cette variété. En effet, l'histoire sociale du breton fait qu'il existe depuis un siècle maintenant ${ }^{33}$, une forme nouvelle et standardisée de breton. Par conséquent, des locuteurs peuvent avoir eu cette langue comme langue première. C'est le cas de Mme Louarn. Dès lors, il est difficile de créer un groupe clairement désigné comme celui des «néo-locuteurs » car ce qui les définit précisément c'est la variété dont ils font usage. Il est vrai que dans ce groupe linguistique Lena Louarn fait un peu figure d'exception pour le mode d'apprentissage, celui-ci résultant d'une transmission familiale. En réalité, pour l'immense majorité de ce groupe, le breton est une seconde langue apprise soit à l'école, soit à l'âge adulte. Ainsi, cet apprentissage résulte du choix délibéré d'un adulte, il est le fruit d'un effort conscient ${ }^{34}$. C'est ce qu'a entrepris le député Paul Molac. Mais cela peut-être aussi la volonté de parents mettant en place une politique linguistique familiale. Cette catégorie a systématiquement une image positive de la langue quand il s'agit d'un apprentissage à l'âge adulte. C'est sans doute moins évident de la part des élèves scolarisés dans les filières bilingues.

\section{Lena Louarn :}

Lors de notre entretien, la vice-présidente déclare parler un breton héritier d'un apprentissage livresque. Ses parents qui lui ont transmis

32. E. LE PIPEC, op. cit., p. 104.

33. Jean LE DÛ, Yves LE BERRE, op. cit., p. 49.

34. Michel Bert, Colette Grinevald, op. cit., p. 129. 
la langue n'étaient pas eux-mêmes bretonnants de première langue. De fait, il est difficile de rattacher la langue parlée par Lena Louarn à un terroir. C'est un idiome exogène qui cherche des racines. On peut constater une volonté de sa part de rattacher son breton à une aire dialectale, du moins aux bretons populaires.

Ainsi, voici quelques faits de langue permettant de rattacher son breton au Nord-Finistère ou au Cap-Sizun dont sa famille maternelle est originaire : elle utilise systématiquement le possessif va. De plus, on peut noter la prononciation en [ou] du phonème [on] : 1.250 et 1.325 Bro-Leoun. Et encore, l'adjectif [spontus] prononcé [spountus] 1.365 et 369. Ou alors, la prononciation du [z] en [ch], pour laquelle je relève plusieurs occurrences : menochoù pour menozioù l. 428.

Ensuite, témoignant d'une volonté de parer son breton d'éléments issus du « breton populaire » on relève, à plusieurs occurrences, l'emploi de l'emprunt au français [tout], théoriquement mal vu parmi les zélateurs du breton unifié. De plus, je relève quelques élisions, propres à certains parlers locaux : 1.91 [dimeet] qui s'écrit et se prononce en breton standard dimezet.

À noter que cette prononciation est plutôt en cours dans les parlers du Sud-Finistère, alors que j'avais le sentiment qu'elle cherchait à inscrire sa langue dans la zone Nord-Finistère. Enfin, on observe l'insertion de mots français, un usage en général prohibé par les mêmes thuriféraires du peurunvan. Voyons quelques exemples : -1.24, [mais], [enfin] à plusieurs reprises et des emprunts en français bretonisé comme en breton paritaire : [chikana] 1.410, ou encore rejionalision 1.456, alors que L'Office de la langue bretonne, dont la présidence est toujours assurée par Mme Louarn, préconise le terme rannvroeladur.

Tout ce qui précède fait état d'un léger tropisme pour les formes non standards de breton. Ainsi, selon Ronan Calvez, aux yeux des promoteurs de ce breton normalisé, son existence même ne se justifie que par celle des formes dialectales bénéficiant d'une légitimité populaire.

Outre ces tiraillements idéologiques, le caractère standardisé du breton de Lena Louarn s'explique par quelques éléments biographiques. Il faut avoir à l'esprit qu'elle n'a jamais vécu à l'intérieur de la zone de pratique traditionnelle du breton, la Basse-Bretagne. Elle a grandi à Villeneuve-Saint-Georges, en région parisienne, avant de suivre ses parents à Rennes. Une ville dans laquelle elle vit toujours depuis. 
En examinant la transcription de notre entrevue, le caractère standard du breton de Mme Louarn apparait assez clairement. La langue parlée par Lena Louarn peut être associée au type «néo-breton».

C'est une langue riche en néologismes. Le lexique fait le moins d'emprunts possibles au français ou au substrat latin. Le but affiché est bien celui que l'on retrouve dans les préconisations de l'Office public de la langue bretonne en matière de signalisation. La « déviance maximale ${ }^{35} »$ dans l'espace public face au français devient la norme.

Voici quelques exemples :

\section{Lexicaux}

- kadaarnat (1.9) pour renforcer.

- kendrec'bin (1.10) pour convaincre. Je n'ai jamais entendu ce verbe dans la bouche d'un locuteur traditionnel. Francis Favereau le perçoit comme un composé de création récente ; provenant sans doute du travail de Le Gonidec ou Francis Vallée.

- delwenn (1.79) pour statue, un emprunt au gallois dans la plus pure tradition de ce «néo-breton ».

- kuzul-mera (1.211) pour conseil d'administration.

- dizalc'bourez. (1.510) : pour indépendantisme.

Le changement sémantique :

- « aoza kentelioù » pour « organiser, proposer des leçons ».

- koumananti pour «abonner».

Une structure syntaxique issue directement du français :

- "d'am soñj on evel Asterix on konezet e-barz.»1.109. Je pense que je suis comme Astérix, je suis tombée dedans].

- "ha bremañ ne vez ket graet mui un eveziadenn da skwer perak ne gomprenan nitra a tout pa vez eur gozeadenn etre daou zen hag a oar brezoneg» 1.351 .36 : [Et maintenant il n'y a plus de remarques par exemple « je ne comprends rien » quand il y a une conversation en breton].

Utiliser Eveziadenn pour « remarque » est typique du néo-breton. Cette occurrence est inusitée en breton dialectal.

- " rediet omp da gaout ur skipaih a c'bellfe respont pe e brezoneg pe skrivfe e bezoneg» 1.41 [Nous sommes obligés d'avoir une équipe qui pour- 
rait répondre en breton]. La forme rediet n'est pas mentionnée dans le dictionnaire de Francis Favereau. L'occurrence la plus employée chez les « bretonnants de tradition » est « oblijet», mais cet emprunt au français est à proscrire dans le néo-breton de Mme Louarn.

\section{Des locuteurs intermédiaires}

En terme sociolinguistique, le cas d'Yvon Abiven est remarquable. Le breton était la langue de la famille. Son année de naissance (1948) le situe dans la dernière génération ayant eu le breton comme première langue. Il précise toutefois qu'il a été élevé dans les deux langues, breton et français. Il grandit dans une communauté rurale - PlounéourTrez - où s'opérait le basculement linguistique. À ce propos, il relève dans l'entretien que les enfants du bourg parlaient déjà le français tandis que lui n'entendait que du breton dans la ferme familiale. C'est sa trajectoire individuelle qui le distingue comme locuteur singulier. Ainsi, s'il est héritier d'un breton dialectal («eur pagan», affirme-t-il), il est aussi un homme éduqué qui s'est intéressé de près à sa langue natale, jusqu'à en acquérir la maîtrise orale et écrite. Par la suite, il devient un militant connu de l'Emsao, le mouvement politique et culturel breton. Cette facette de sa personnalité se fait moins prégnante avec le temps alors qu'il se désengage de Diwan et rompt avec l'UDB. Il connait donc différentes variétés de breton, le breton hérité, le néo-breton. Mais de quel breton fait-il usage?

M. Abiven vit depuis trois décennies à Saint-Thégonnec, commune dont il était le maire pendant vingt-cinq ans. Il explique volontiers que le fait d'être bretonnant l'a aidé à se faire connaître lors de son implantation dans la commune. À travers son breton, ses futurs administrés l'identifient comme extérieur à la paroisse. Il est intéressant de chercher à comprendre dans quelle mesure sa langue est restée celle de son enfance, le parler de Plounéour-Trez. J'ai réalisé une modeste étude de dialectologie à travers trois exemples :

- la locution verbale «j'étais en train », Yvon Abiven a recours systématiquement à la forme [édŏn]. Cette forme est attestée dans l'atlas linguistique de Pierre Le Roux au point 4 qui correspond à la commune de Saint-Frégant non loin (huit kilomètres) de Plouneour-Trez. Si l'on trace une isoglosse la commune de 
Plouneour-Trez se trouve à l'intérieur. $A$ contrario, le point 12 qui concerne la commune de Sainte-Sève relève [é oăn]. SaintThégonnec commune limitrophe de Sainte-Sève ne connait probablement pas les formes proches de [édŏn].

- Une «journée» M. Abiven utilise ['derves] pour «journée». C'est ce qu'on relève au point 15 du NALBB $^{36}$ qui correspond à la commune de Plounéour-Trez. Ce rothacisme ne s'observe que dans une zone limitée au nord-ouest du Finistère. SaintThégonnec se trouve largement en dehors de cette zone.

- «ferme» M. Abiven utilise ['atãn] pour désigner la ferme. On trouve cette désinence au point 18 non loin de Plounéour-Trez. Si on trace une isoglosse, la zone de Saint-Thégonnec en est exclue.

Le parler d'Yvon Abiven est resté assez proche de celui de sa prime enfance. Le lexique l'atteste, ainsi que la prononciation de certaines formes verbales inconnues à Saint-Thégonnec.

L'entretien assez long que j'ai pu avoir avec lui fait apparaitre une utilisation parcimonieuse du lexique néo-breton. Voici quelques exemples :

Il utilise kevredigezpour « association » ou kuzulier pour « conseiller». Il connait le champ lexical de l'enseignement (ce n'est pas une surprise, il a exercé ce métier pendant toute sa vie professionnelle) : deskadurez. stad, pour "Éducation nationale », skolaj pour " collège », skol-vamm pour « école maternelle », tenzorier pour « trésorier ». Je relève un nombre significatif d'occurrences provenant du français (comme nous l'avons vu, une des caractéristiques du néo-breton c'est de chercher à s'en éloigner le plus possible). Ainsi, il utilise « député », à la place de kannad, le terme intronisé en "néo-breton ». Il fait usage également des participes passés suivant: Aksepted, entred, en em bresanted, formes bretonisées de verbes français. Le recours systématique à «komañset» montre que son breton est plus proche des parlers hérités que du « néobreton ». Ce dernier lui préfère toujours kregiñ, pour se distancier au maximum du français.

36. Jean LE DÛ, op. cit. 
L'ancien maire de Saint-Thégonnec se singularise d'un point de vue linguistique. Il parle une variété dialectale assez proche de celle de son enfance. Il connait les néologismes, mais n'en use qu'avec parcimonie.

Le breton dont fait usage Didier Goubil le rattache à la catégorie des semi-locuteurs. Néanmoins, il est également proche du type des locuteurs de tradition. Ainsi, M. Goubil a appris le breton au sein de la ferme familiale. Celle-ci abritait également ses grands-parents qui s'adressaient à lui en breton. Le breton dont il a l'usage est bien hérité du breton vernaculaire de cette paroisse.

Néanmoins, j'ai choisi de le placer dans cette catégorie des semi-locuteurs car son breton est émaillé de fautes importantes qui démontrent une acquisition inachevée ou imparfaite de la langue.

Voici quelques exemples qui illustrent cet état de fait :

- On note l'absence systématique de participe passé, l'infinitif est toujours employé en lieu et place du participe passé :

- "kozel meum in galleg, nann nann kozelpeus pad tout al lein in brezoneg, me meu meus ke grêt intansin kwa, pengur, oagn ko oam oam kozel deus o bern traoù, a ve kozel in brezoneg, sed a giz-s, ba me choñj voien zo deus kozel in brezoneg »

- Ou encore, les mutations consonantiques non réalisées :

- "kap deu deu kozel en brezoneg»

- "pe meus komans de kozel»

- «me koze en brezon»

- Ou alors, un recours à des termes français pour des mots usités en breton vernaculaire :

- "n’heign ket gwall, o problème santé », je dois lui rappeler que santé ce dit yec'hed en breton

- «vênt gênés"

- «tout an traoù a zo, zo ordinaires »

- « ha utilise noñ o vad»

Ces éléments font apparaittre une attrition linguiste ${ }^{37} \mathrm{du}$ breton de M. Goubil. C'est bien ce que recouvre ce terme pour Barbara Kopke : «L'attrition fait référence à la perte d'éléments structurant de

37. Barbara KOPKE, Monika SCHMIDT, «L'attrition de la première langue en tant que phénomène psycholinguistique », Language. Interaction and Acquisition, 2011, p. 197-220. 
la langue, c'est-à-dire à une modification ou à une réduction de la forme linguistique. » Cependant, un élément majeur suffit à replacer le maire de Poullaouen parmi les locuteurs de tradition. C'est une pratique courante, quotidienne, du breton qui le rattache aux locuteurs de tradition. Selon M. Goubil, les occasions de pratiquer la langue ne manquent pas dans ce coin du Centre-Bretagne. Comme exemples, il mentionne les sorties de chasse entre amis, le fait de couper une pièce de bois, ou encore de s'atteler à un travail manuel. La fluidité du discours de Didier Goubil démontre clairement une pratique courante du breton. D'évidence, il est exposé quotidiennement au breton dans sa commune et bien que sa langue soit frappée par une certaine attrition, son aisance à l'oral est incontestable. De fait, notre entretien a été mené tambour battant sans beaucoup d'interruptions de la part du maire. En outre, je relève des traits dialectaux très marqués qui confirment que le maire de Poullaouen est à cheval entre deux types de locuteurs :

Voici quelques exemples :

\section{Lexicaux}

- Ferme : [feurm] c'est le vocable relevé par Francis Favereau, très bon connaisseur du parler de Poullaouen.

- Jeune : [yaoun]

- Breton : [brezon]

Construire un objet de recherche à partir d'une enquête de terrain représente un défi passionnant. Il est difficile d'esquiver certaines difficultés méthodologiques. D’une part, on s'expose aux imprévus liés aux interactions chercheur-témoin. Dans ce cadre, l'objectivité sacro-sainte enseignée dans l'institution universitaire est une gageure. À moins de réifier l'objet-témoin, il est difficile de s'abstraire de phénomène d'empathie ou de sentiments contraires. D'autre part, la construction de groupes linguistiques peut sembler, dans sa réalisation, assez arbitraire. Ainsi, certains témoins peuvent appartenir à plusieurs groupes, une typologie de locuteurs est une construction artificielle. Mis à part ces quelques obstacles, travailler à partir du matériau brut de la parole humaine, s'avère, dans les domaines de la sociolinguistique ou de la science politique une aventure très enrichissante et pourvoyeuse de sens pour le chercheur débutant. 